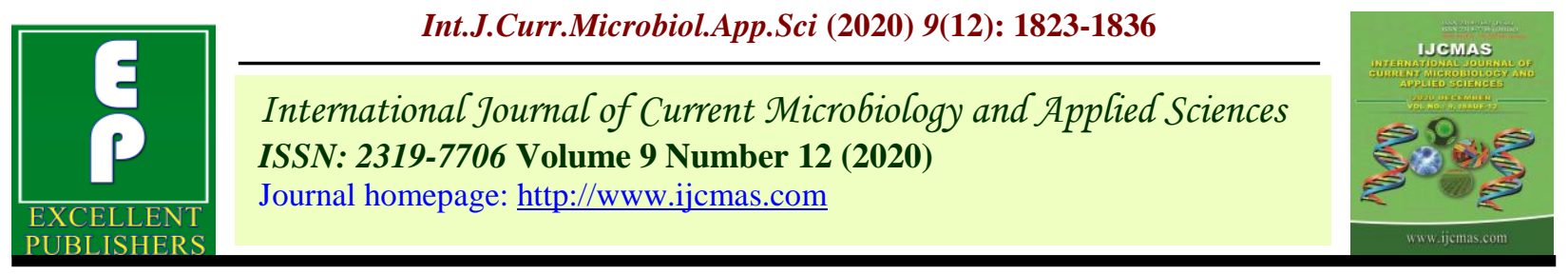

Original Research Article

https://doi.org/10.20546/ijcmas.2020.912.217

\title{
Impact of Flowering Stage Drought Stress on Yield and Yield Related Attributes on Rice (Oryza sativa L) Genotypes
}

\author{
Arun Kumar ${ }^{1 *}$, R. S. Sengar ${ }^{1}$, Manoj Yadav, Shalani Gupta², \\ Ramji Singh $^{3}$ and Pooran Chand ${ }^{4}$ \\ ${ }^{1}$ Department of Ag. Biotechnology, ${ }^{2}$ Department of Molecular Biology \& Genetic \\ Engineering, ${ }^{3}$ Department of Plant Pathology, ${ }^{4}$ Department of Genetics and Plant Breeding, \\ Sardar Vallabhbhai Patel University of Agriculture and Technology, \\ Modipuram, Meerut, India-250110 \\ *Corresponding author
}

\section{A B S T R A C T}

Rice is a staple food crop in India as well as whole world. Plant growth and productivity of rice is adversely affected by various biotic and abiotic stress factors. Water deficit is one of

\section{Keywords}

Rice, Water stress, Morphological, Yield attributes

Article Info

Accepted:

14 November 2020

Available Online:

10 December 2020 the major abiotic stresses, which adversely affects crop growth and yield. Water stress is a limiting factor in agriculture production by preventing a crop from reaching the genetically determined theoretical maximum yield. In present study, a pot experiment was conducted with 20 rice (1 Non Basmati and 19 Basmati) genotypes during kharif season 2018-19 and 2019-20 at field laboratory, Department of Agricultural Biotechnology, S.V.P.U.A.\&T., Modipuram, Meerut, U.P., India in Completely Randomized Design (CRD) with three replications. Drought treatment was given for 10 days at flowering stage. The screening of rice genotypes was done on the basis of plant height, panicle length, seed per panicle, yield per plant, test weight and flag leaf area. The rice genotypes Nagina22 followed by Pusa Basmati 1121 showed less percent reduction in yield and yield components comparatively all other varieties. The maximum reduction in yield and other parameters under drought stress condition was observed in Basmati 386 variety. Therefore, on the basis of yield and yield component Nagina 22 showed maximum tolerance in compare to other genotypes.

\section{Introduction}

Rice (Oryza sativa L.) is an important food crop in India and several parts of the globe. It is the second most cultivated cereal in the world and most widely consumed staple food for about $60 \%$ of the world's population (Pirdashti et al., 2009) and occupied 197.59 million hectare area in the world with 996.07 million tones production (FAOSTAT, 201819). It shares maximum in grain production and occupies largest area under cultivation in India. India ranks second in rice production across the world and produced over 172.58 million tons during the crop year 2018-19 and occupies 44.5 million hectare area of harvesting (FAOSTAT, 2018-19). India is the largest rice growing country, while china is the largest producer of rice. In India, it accounts for more than $40 \%$ of food grain production, providing direct employment to $70 \%$ people in rural areas. Being the staple 
food for more than $65 \%$ of the people, our national food security hangs on the growth and stability of its production. The key goals of varietal development are yield, quality characteristics and biotic/abiotic stress tolerance (Nachimuthu et al., 2015).

With the increasing population, the demand of rice will going to increase day by day. So, to meet the global rice demand, its production needs to be increase (Khush, 2005). Plants respond to various abiotic factors like drought, high salt, heavy metals, changes in temperature and light in the environment. Abiotic stresses affect the plant at morphological, physiological, biochemical and molecular level. Several abiotic stresses like Drought, high temperatures, salinity and oxidative stress are often interrelated, and may cause similar changes in plants (Szekeres et al., 2003). The phenomenon of insufficient water supply to plants is known as Drought. It limits the crop production and is becoming a gradually more severe problem in many regions of the globe (Passioura, 1996, 2007). As rice is an aquatic plant hence it is very sensitive to drought stress for almost all growth phases which leads to drastic reduction in grain yield (O'Toole, 1982, Hsiao et al., 1984, Venuprasad et al., 2008, Bouman et al., 2005).

Low moisture condition at flowering is most common and serious concern in upland rice ecosystem. The yield of upland rice is highly inconsistant because of drought especially at flowering stage. Drought at flowering stage is very destructive and sometimes it reduce the $70-80 \%$ yield. A lot of work had been already done on drought resistance in rice at vegetative stage (Yang et al., 2014) but research work on impact of drought stress in rice at flowering stage is limited. Therefore, there is requirement to identify excellent donors of upland rice tolerant to drought at flowering stage (Chaturvedi and Ingram, 1988; Mishra, 2005). Like other abiotic stresses, Initially drought affects water relations on the cellular level as well as entire plant (Beck et al., 2007). It impacts on several physiological, biochemical and molecular processes, like ion uptake, translocation, respiration, photosynthesis, carbohydrates, nutrient metabolism, harmonic balance and level of expression of several genes (Farooq et al., 2009b). This results to the induced production of stress proteins and accumulation of compatible solutes by these diverse environmental stresses cause of cellular responses and cell signaling pathways of a plant. During changing environment scenario plants go through certain biochemical adaptations to sustain by evolution of new metabolic pathways which resulted to changes in biochemistry of the cell (Fujita et al., 2006).

Several studies have shown that drought tolerance is a very complex feature, as it is a combined feature of a number of morphological, physiological and biochemical characteristics (Mishra, 2005, Pandey and Shukla, 2015).

To characterize the genotypic performance of rice under drought stress, plant height, panicle number, root volume, fertile spikelets, plant biomass, leaf area development, root/shoot ratio, grain yield, chlorophyll, starch, soluble sugar and proline contents were used in various drought stress experiments (Mishra, 2005; Maisura et al., 2014). Hence, keeping above considerations in mind the present investigation was carried out to determine the impact of drought stress at flowering stage in rice varieties on morphological components.

\section{Materials and Methods}

Experiment location, plant materials, and observation of phenotypic trait

The present experiment was conducted during kharif-2018 and kharif-2019 consists 
of 20 (19 Basmati and 1 Non-Basmati) rice varieties, which were collected from BEDF Meerut, India and Zonal research station Nagina, Bijnor, India (Table 1). After 21 days of seedling emergence, the seedling were transplanted in pots $\left(39 \times 33 \mathrm{~cm}^{2}\right.$ pot size containing $25 \mathrm{Kg}$ of soil and $5 \mathrm{Kg}$ of FYM) at Experimental plot, Department of Agricultural Biotechnology, S.V.P.U.A.\&T., Modipuram, Meerut, U.P., India, which is situated at $26.47^{\circ} \mathrm{N}$ (latitude), $82.12^{\circ} \mathrm{E}$ (longitude) and at $113 \mathrm{~m}$ above mean sea level in Completely Randomized Design (CRD) with three replications. The soil of experimental site was sandy loam with initial pH.-7.2 and $\mathrm{EC}_{\mathrm{e}}$ of $1.39 \mathrm{dSm}^{-1}$. Recommended dose of fertilizer at the rate N: P: K 100:40:40 kg/ha on the pot area basis. After 30 days of planting, five plants were maintained in each pot by thinning process. Water deficit was created for 10 days at $50 \%$ flowering by withholding irrigation in pots. However, control pots were frequently irrigated to optimum field capacity level.

\section{Weather conditions}

The meteorological observations were recorded by an automatic weather station of Indian Institute of Farming System and Research (IIFSR), Modipuram, Meerut, India. Minimum and maximum temperature, percentage of relative humidity in morning and evening, average rainfall and bright sunshine were observed for the month from June- Nov 2018 and from June- Nov 2019 (Table 2 and Graph 1).

\section{Observation of phenotypic trait}

Morphological traits were recorded after stress treatment in drought stressed and control plants. The traits viz., plant height, number of tillers, panicle length, seed per panicle, yield per plant, 1000 seed weight and leaf area were recorded for three randomly selected plants for each replication for phenotypic trait analysis. Plant height was measured in $\mathrm{cm}$ from the base of the shoot to the tip of the main stem at ripening stage and averaged over three plants (O' Toole and Cruz, 1983). Whereas at the ripening point, the panicle length was measured in $\mathrm{cm}$ from the panicle neck to the tip and averaged over three plants and a healthy panicle was taken for counting the number of grains and counted at the time of harvesting in a per plant then averaged over three plants. For the grains of each randomly chosen plant, hand threshing was carried out then sun dried and weighed in grams. By weighing 1000 filled grains, the test weight was recorded in grams and averaged over three samples for each variety. Leaf areas were calculated by using index leaf method given by Sticker et al., (1961). As, leaf area $=\mathrm{L}^{*} \mathrm{~W}^{*} \mathrm{~F}$, where, $\mathrm{L}=$ Maximum length $(\mathrm{cm}), \mathrm{W}=$ maximum width (cm), F= Factor (0.70).

\section{Statistical analysis}

Data presented are mean of three replicates. The data were subjected to ANOVA by OPSTAT software. The differences at $(p \leq$ $0.05)$ were considered as significant.

\section{Results and Discussion}

\section{Weather observations}

The mean of meteorological variables data are presented (Table 2 and Graph 1) during the experimental period (June-Nov. 2018 and 2019). During the whole experimental period, weekly min and max temperature ranged from $11.78^{\circ} \mathrm{C}$ to $27.53^{\circ} \mathrm{C}$ with a general mean of $19.65{ }^{\circ} \mathrm{C}$ and 29.10 to 41.7 with average mean of $35.4{ }^{\circ} \mathrm{C}$. Total rainfall received was ranged from $0.0 \mathrm{~mm}$ to $323.10 \mathrm{~mm}$, relative humidity varies from $82.75 \%$ to $95.88 \%$ in morning whereas in evening time it varied from $45.75 \%$ to 
$72.10 \%$ and $121.99 \mathrm{~h}$ of bright sunshine (BSS) during entire experimental period.

\section{Effect of drought stress at flowering stage on morphological traits}

\section{Plant height}

Drought stress significantly affected mean plant height in all the twenty genotypes for year 2018-19 and 2019-20. In year 2018-19, the results showed that the plant height under irrigated condition varied from a lower value of $92.0 \mathrm{~cm}$ (Pusa Basmati 6) to higher value of $128.90 \mathrm{~cm}$ (Tarori basmati) while under simulated drought condition, it was reduced significantly and varied from a lower value of $87.60 \mathrm{~cm}$ (Punjab Basmati 4) to $118.33 \mathrm{~cm}$ (Taraori Basmati) (Table 3). The minimum reduction was observed in genotype Nagina 22 (2.71\%) followed by genotype Pusa Basmati $1121(3.33 \%)$ and the maximum difference was observed in genotype Basmati 386 (8.90\%) (Graph 2).

In year 2019-20, the plant height under irrigated condition varied from a lower value of $92.5 \mathrm{~cm}$ (Pusa Basmati 6) to higher value of $129.5 \mathrm{~cm}$ (Tarori Basmati) while under simulated drought condition, it was reduced significantly and varied from a lower value of $92.10 \mathrm{~cm}$ (Vallabh Basmati 22) to $118.73 \mathrm{~cm}$ (Tarori Basmati) (Table 4). The minimum difference was observed in genotype Nagina $22(2.31 \%)$ followed by genotype Pusa Basmati-1121 (3.47\%) and maximum difference was observed in genotype Basmati 364 (9.31\%) (Graph 3).

\section{Panicle length}

In year 2018-19, the results showed that the panicle length under irrigated condition varied from a lower value of $20.5 \mathrm{~cm}(\mathrm{~N} \mathrm{22})$ to higher value of 33.13 (Pant Basmati 2). Under simulated drought condition, it was reduced significantly and varied from a lower value of $19.03 \mathrm{~cm}$ (Nagina 22) to $30.63 \mathrm{~cm}$ (Basmati 564) (Table 3). The minimum difference was observed in genotype Nagina $22(7.16 \%)$ followed by genotype Pusa Basmati 1121 (7.23\%) and the maximum difference was observed in genotype Basmati 386 (15.69\%) (Graph 2).

In year 2019-20, the results showed that the panicle length under irrigated condition varied from a lower value of $20.3 \mathrm{~cm}$ (Nagina 22) to higher value of $34.73 \mathrm{~cm}$ (Basmati 564). Under simulated drought condition, the panicle length was reduced significantly and varied from a lower value of $18.86 \mathrm{~cm}$ (Nagina 22) to 30.1 (Basmati 564) (Table 4). The minimum difference was observed in genotype Nagina 22 (7.06\%), followed by genotype Pusa Basmati 1121 (8.54\%) and the maximum difference was observed in genotype Basmati 386 (13.51\%).

\section{Seed per panicle}

In year 2018-19, the results showed that the seed per panicle under irrigated condition varied from a lower value of 97 (Pusa Basmati 1509) to higher value of 216.33 (Pant Basmati 2). Under simulated drought condition, the panicle length was reduced significantly and varied from a lower value of 57.70 (Basmati 386) to 129.33 (Pant Basmati 2) (Table 3). The minimum difference was observed in genotype Nagina 22 (14.74\%) followed by genotype Pusa Basmati 1121 $(22.83 \%)$ and the maximum difference was observed in genotype Basmati 386 (46.56\%) (Graph 2).

In year 2019-20, the results showed that the seed per panicle under irrigated condition varied from a lower value of 95.33 (Pusa Basmati 1509) to higher value of 215.33 (Pant Basmati 2). Under simulated drought condition, the seed per panicle was reduced significantly and varied from a lower value of 57 (Basmati 386) to 128.66 (Pant Basmati 2) 
(Table 4). The minimum difference was observed in N $22(15.37 \%)$ followed by Pusa Basmati 1121 (22.26\%) and the maximum difference was observed in genotype Basmati $386(45.71 \%)$.

\section{Grain yield per plant under irrigated and drought condition}

Yield and yield characteristics are the ultimate manifestation of the ability of a plant to sustain growth and produce yield under water, regardless of the resistance processes involved. In year 2018-19, the values of grain yield ranges between $7.78 \mathrm{gm}$ (Basmati 386) to 21.63 gm (Pant Basmati 1). Under drought condition, the grain yield range was decreased to 2.97 gm (Basmati386) (lowest) and 14.13 gm (Pusa Basmati 1121) (highest) (Table 3). The minimum difference in yield was observed Nagina 22 (12.32\%) followed Pusa Basmati 1121 $(14.91 \%)$ and the maximum difference was observed in genotype Basmati 386 (61.83\%) (Graph 2).

While in the year 2019-20, controlled plants of 20 genotypes showed a grain yield from 7.82 gm (Basmati 386) to $21.66 \mathrm{gm}$ (Pant Basmati 1). However, grain yield under stressed condition varied from a lower value of $3.10 \mathrm{gm}$ (Basmati 386) to a higher value of $13.8 \mathrm{gm}$ in (Pusa Basmati 1121) (Table 4). The average difference in grain yield under irrigated and non-irrigated condition was observed minimum in genotype $\mathrm{N}-22$ (12.03\%), followed by Pusa Basmati-1121 $(15.01 \%)$ and the maximum difference was observed in Basmati 386 (60.32\%).

\section{Test weight (1000 grain weight)}

In year 2018-19, the results showed that the test weight under irrigated condition varied from a lower value of $19.93 \mathrm{gm}$ in genotype (Vallabh Basmati 23) to higher value of 28.5 gm (Pusa Basmati 1609). Under simulated drought condition the test weight was reduced significantly and varied from a lower value of $14.68 \mathrm{gm}$ (Vallabh Basmati 23) to 22.69 gm (Pusa Basmati 1121) (Table 3). The minimum difference was observed in genotype Nagina $22(8.46 \%)$ followed by Pusa Basmati $1121 \quad(15.21 \%)$ and the minimum difference was observed in genotype Basmati 386 (30.6\%) (Graph 2).

In year 2019-20, the results showed that test weight under irrigated condition varied from a lower value of 20.21 gm (Vallabh Basmai 23 ) to higher value of $28.70 \mathrm{gm}$ (Pusa Basmati 1609). Under simulated drought condition the test weight was reduced significantly and varied from a lower value of 14.80 gm (Vallabh Basmati 23) to $22.65 \mathrm{gm}$ (Pusa Basmati 1121) (Table 4). The minimum difference was observed in genotype Nagina $22(9.35 \%)$ followed by moderate in genotype Pusa Basmati 1121 $(15.33 \%)$ and maximum difference was observed in genotype Basmati 386 (31.66\%) (Graph 3).

\section{Effect of drought on leaf area}

Leaf area is directly related to increased photosynthesis and chlorophyll content. Therefore, this is a crucial factor for understanding the proper growth and development of plant. Drought stress significantly affected mean leaf area $\left(\mathrm{cm}^{2}\right)$ in all twenty rice genotypes.

In year 2018-19, the results showed that the leaf area under irrigated condition varied from a lower value of $25.2 \mathrm{~cm}^{2}$ (Punjab Basmati 4) to higher value of $54.23 \mathrm{~cm}^{2}$ (Pusa Basmati 1609). Under simulated drought condition, the leaf area was reduced significantly and varied from a lower value of $17.03 \mathrm{~cm}^{2}$ (Basmati 386) to $40.8 \mathrm{~cm}^{2}$ (Punjab Basmati 2) (Table 3). The minimum difference was observed in genotype $\mathrm{N}-22$ (13.26\%) followed by genotype Pusa Basmati 
$1121(17.83 \%)$ and the maximum difference was observed in genotype Basmati 386 (35.24\%) (Graph 2).

In year 2019-20, the results showed that the leaf area under irrigated condition varied from a lower value of $25.2 \mathrm{~cm}^{2}$ (Tarori Basmati) to higher value of $54.5 \mathrm{~cm}^{2}$ (Pusa Basmati 1609). Under simulated drought condition, the leaf area was reduced significantly and varied from a lower value of $16.63 \mathrm{~cm}^{2}$ (Tarori Basmati) to $40.73 \mathrm{~cm}^{2}$ (Pusa Basmati 1609) (Table 4). The minimum difference was observed in $\mathrm{N}-22$ (14.79\%) followed by Pusa Basmati 1121 $(17.96 \%)$ and the maximum difference was observed in genotype Basmati 386 (34.68\%) (Graph 3).

Table.1 Description of different rice genotypes used in present study

\begin{tabular}{|c|c|c|c|}
\hline Genotype name & Origen & Pedigree & $\begin{array}{l}\text { Releasing } \\
\text { year }\end{array}$ \\
\hline Nagina 22 & Nagina, U P, India & - & 1978 \\
\hline Panjab Basmati 04 & PAU, India & - & 2017 \\
\hline Panjab Basmati 02 & PAU, India & - & 2012 \\
\hline Pusa basmati 1609 & IARI, New Delhi, India & $\begin{array}{l}\text { Elite Basmati restorer line } \\
\text { PRR78/C101A51 }\end{array}$ & 2015 \\
\hline Vallabh Basmati 23 & SVBPUA \& T, India & - & 2015 \\
\hline Pusa Basmati 1 (IET 10364) & IARI, New Delhi, India & Pusa150/Karnal Local & 1989 \\
\hline $\begin{array}{l}\text { Pusa Basmati } 1509 \text { (IET } \\
\text { 21960) }\end{array}$ & IARI, New Delhi, India & Pusa Basmati 1121/ Pusa 1301 & 2013 \\
\hline $\begin{array}{l}\text { Tarori Basmati (HBC-19 or } \\
\text { Karnal local) }\end{array}$ & HAU, Kaul, India & Selection from Basmati 370 & 1996 \\
\hline Basmati 564 & SKUAST- Jammu, India & - & 2015 \\
\hline Pusa Basmati 6 & IARI, New Delhi, India & Pusa Basmati 1/1121 & 2010 \\
\hline $\begin{array}{l}\text { Vallabh Basmati } 21 \text { (IET } \\
\text { 19493) }\end{array}$ & SVBPUA \& $\mathrm{T}$, India & - & 2013 \\
\hline Basmati CSR 30 & CSSRI, Karnal, India & Buraratha 4-10/ Pakistani Basmati & 2012 \\
\hline Vallabh Basmati 24 & SVBPUA \& T, India & - & 2015 \\
\hline Type 3 or Dehradun Basmati & Nagina, U P, India & Selection from Basmati Deharadun & 1978 \\
\hline Basmati 386 & PAU, Punjab, India & Selection from Pakistani Basmati & 1994 \\
\hline Pant Basmati 2 (IET 21953) & GBPUA\&T,U.K,India & - & 2016 \\
\hline Pusa Basmati 1121 & IARI, New Delhi, India & Sister line of Pusa Basmati-1 & 2008 \\
\hline Pant Basmati 1 (IET 21665) & GBPUA\&T,U.K,India & Pusa Basmati 1/ IET 12603 & 2016 \\
\hline Pusa sugandha 5 & IARI, New Delhi, India & Pusa 3 A 9 Haryana Basmati & 2005 \\
\hline Vallabh Basmati 22 & SVBPUA \&T, India & - & 2009 \\
\hline
\end{tabular}


Table.2 Standard meteorological variables during the months of June-Nov. 2018 and 2019

\begin{tabular}{|c|c|c|c|c|c|c|c|}
\hline Week & $\begin{array}{c}\text { Temp. } \\
\max .\left({ }^{\circ} \mathrm{C}\right)\end{array}$ & $\begin{array}{l}\text { Temp. } \\
\text { min. }\left({ }^{\circ} \mathrm{C}\right)\end{array}$ & $\begin{array}{c}\text { RH } \\
\text { morning( } \%)\end{array}$ & $\begin{array}{c}\text { RH } \\
\text { evening }(\%)\end{array}$ & WS (km/hr) & BSS (hrs) & $\begin{array}{c}\text { Rainfall } \\
\text { (mm) }\end{array}$ \\
\hline 25 & 41.70 & 26.90 & 82.75 & 45.75 & 1.95 & 7.71 & 0.00 \\
\hline 26 & 39.25 & 27.53 & 83.31 & 56.80 & 3.70 & 6.40 & 6.75 \\
\hline 27 & 38.90 & 25.88 & 89.15 & 51.95 & 2.44 & 6.67 & 41.63 \\
\hline 28 & 34.92 & 26.73 & 90.28 & 72.10 & 2.41 & 2.90 & 73.18 \\
\hline 29 & 36.07 & 27.19 & 88.59 & 57.25 & 1.83 & 5.08 & 16.30 \\
\hline 30 & 33.40 & 26.05 & 93.28 & 70.73 & 1.60 & 3.56 & 323.10 \\
\hline 31 & 36.52 & 27.20 & 91.43 & 55.61 & 1.69 & 4.06 & 16.48 \\
\hline 32 & 36.00 & 26.76 & 94.00 & 55.37 & 1.79 & 4.20 & 45.30 \\
\hline 33 & 37.04 & 26.78 & 92.86 & 59.50 & 1.64 & 5.53 & 46.05 \\
\hline 34 & 35.73 & 24.80 & 92.25 & 57.28 & 1.50 & 5.53 & 17.55 \\
\hline 35 & 35.25 & 24.00 & 89.55 & 54.20 & 1.75 & 3.23 & 166.15 \\
\hline 36 & 36.80 & 22.63 & 92.50 & 57.70 & 1.53 & 3.10 & 63.40 \\
\hline 37 & 34.80 & 22.98 & 88.73 & 58.13 & 2.30 & 5.73 & 7.28 \\
\hline 38 & 34.70 & 22.23 & 89.28 & 57.45 & 7.25 & 4.90 & 10.33 \\
\hline 39 & 32.28 & 22.90 & 94.20 & 74.85 & 11.18 & 5.85 & 31.35 \\
\hline 40 & 34.73 & 20.68 & 95.88 & 59.35 & 7.00 & 8.45 & 5.73 \\
\hline 41 & 33.55 & 18.75 & 88.63 & 55.50 & 11.43 & 7.10 & 2.55 \\
\hline 42 & 33.80 & 15.88 & 91.18 & 53.50 & 3.63 & 7.00 & 1.05 \\
\hline 43 & 32.05 & 14.03 & 91.50 & 49.55 & 4.95 & 7.13 & 0.00 \\
\hline 44 & 31.23 & 12.95 & 91.75 & 47.48 & 6.80 & 4.95 & 0.00 \\
\hline 45 & 30.13 & 11.83 & 93.68 & 49.48 & 5.55 & 6.78 & 0.00 \\
\hline 46 & 29.10 & 11.78 & 94.78 & 53.50 & 8.00 & 6.18 & 0.00 \\
\hline
\end{tabular}

Note- Temp.: temperature, RH: relative humidity, BSS: bright sun shine, WS: wind speed

Graph.1 Standard meteorological variables during the months of June-Nov. 2018 and 2019

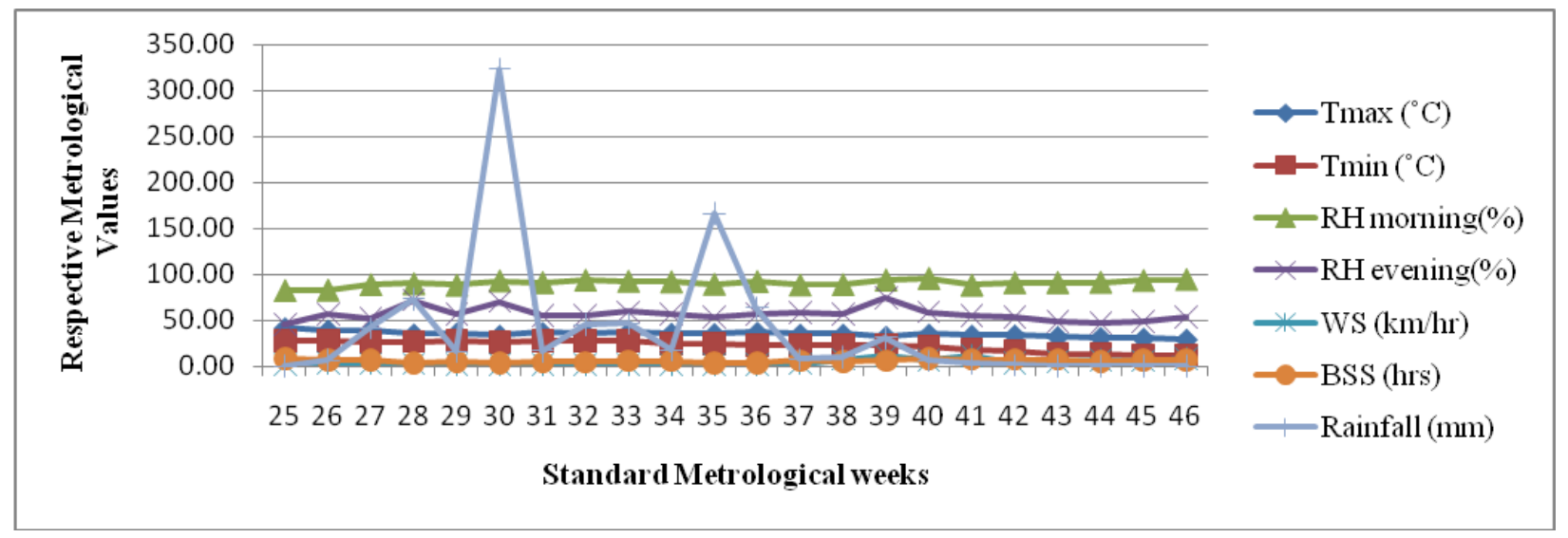


Table.3 Morphological performance of rice genotypes under drought stress (2018-19)

\begin{tabular}{|c|c|c|c|c|c|c|c|c|c|c|c|c|}
\hline \multirow[t]{2}{*}{ Name of variety } & \multicolumn{2}{|c|}{ Plant height } & \multicolumn{2}{|c|}{ Panicle length } & \multicolumn{2}{|c|}{ Seed per panicle } & \multicolumn{2}{|c|}{ Yield per plant } & \multicolumn{2}{|c|}{ Test weight } & \multicolumn{2}{|c|}{ Leaf area } \\
\hline & C & DS & C & DS & $\mathrm{C}$ & DS & C & DS & C & DS & C & DS \\
\hline N 22 & $98.46 \pm 0.72$ & $95.8 \pm 0.37$ & $20.5 \pm 0.28$ & $19.03 \pm 0.63$ & $147 \pm 0.57$ & $125.33 \pm 0.66$ & $14.12 \pm 0.29$ & $12.38 \pm 0.27$ & $22.01 \pm 0.38$ & $20.14 \pm 0.52$ & $29.66 \pm 0.57$ & $25.73 \pm 0.57$ \\
\hline Pnb Bas.4 & $92.58 \pm 0.71$ & $87.6 \pm 0.37$ & $23 \pm 0.28$ & $20.73 \pm 0.28$ & $119 \pm 1.0$ & $85 \pm 0.57$ & $16.44 \pm 0.34$ & $9.083 \pm 0.33$ & $26.23 \pm 0.13$ & $20.02 \pm 0.11$ & $25.2 \pm 0.52$ & $19.46 \pm 0.57$ \\
\hline Pnb Bas. 2 & $112.3 \pm 0.72$ & $104.23 \pm 0.40$ & $29.26 \pm 0.14$ & $25.73 \pm 0.69$ & $109.33 \pm 0.88$ & $67.33 \pm 1.45$ & $11.12 \pm 0.23$ & $5.57 \pm 0.23$ & $24.03 \pm 0.15$ & $17.43 \pm 0.10$ & $31.7 \pm 0.62$ & $23.8 \pm 0.86$ \\
\hline PB. 1609 & $104.66 \pm 0.88$ & $97.96 \pm 0.38$ & $29.9 \pm 0.20$ & $25.8 \pm 0.55$ & $214.33 \pm 0.88$ & $126.33 \pm 0.33$ & $17.36 \pm 0.36$ & $11.83 \pm 0.26$ & $28.5 \pm 0.45$ & $21.30 \pm 0.12$ & $54.23 \pm 0.57$ & $40.8 \pm 0.60$ \\
\hline VB. 23 & $106.73 \pm 0.88$ & $100.03 \pm 0.41$ & $26.33 \pm 0.16$ & $23.9 \pm 0.25$ & $115 \pm 0.577$ & $83.66 \pm 0.33$ & $12.4 \pm 0.26$ & $8.39 \pm 0.23$ & $19.93 \pm 0.41$ & $14.68 \pm 0.14$ & $31.23 \pm 0.50$ & $24.1 \pm 0.63$ \\
\hline PB. 1 & $115.5 \pm 0.41$ & $106.83 \pm 0.46$ & $30 \pm 0.11$ & $26.63 \pm 0.40$ & $179 \pm 1.15$ & $108 \pm 0.57$ & $16.89 \pm 0.35$ & $10.76 \pm 0.22$ & $22.79 \pm 0.42$ & $18.09 \pm 0.10$ & $30.4 \pm 0.60$ & $21.96 \pm 0.54$ \\
\hline PB. 1509 & $97.83 \pm 0.72$ & $92.53 \pm 0.35$ & $26.53 \pm 0.14$ & $23.46 \pm 0.52$ & $97 \pm 0.57$ & $64 \pm 0.57$ & $16.12 \pm 0.33$ & $10.50 \pm 0.22$ & $26.27 \pm 0.14$ & $18.53 \pm 0.10$ & $32.9 \pm 0.55$ & $22.63 \pm 0.66$ \\
\hline Tarori & $128.9 \pm 0.29$ & $118.33 \pm 0.50$ & $28.66 \pm 0.14$ & $25.06 \pm 0.54$ & $118 \pm 1.15$ & $74.33 \pm 0.88$ & $14.61 \pm 0.30$ & $8.66 \pm 0.23$ & $24.66 \pm 0.43$ & $18.35 \pm 0.11$ & $25.56 \pm 0.98$ & $17.13 \pm 0.98$ \\
\hline Bas. 564 & $127.8 \pm 0.30$ & $116.83 \pm 0.50$ & $34.96 \pm 0.26$ & $30.63 \pm 0.76$ & $204.66 \pm 0.88$ & $123.66 \pm 0.33$ & $12.45 \pm 0.26$ & $6.74 \pm 0.32$ & $20.91 \pm 0.43$ & $15.72 \pm 0.21$ & $28.5 \pm 0.60$ & $21.53 \pm 0.54$ \\
\hline PB. 6 & $92 \pm 0.41$ & $87.63 \pm 0.35$ & $28.13 \pm 0.63$ & $24.5 \pm 0.61$ & $154 \pm 0.57$ & $101.33 \pm 0.88$ & $18.4 \pm 0.38$ & $9.23 \pm 0.17$ & $22.08 \pm 0.45$ & $15.43 \pm 0.22$ & $36.3 \pm 0.63$ & $28.46 \pm 0.54$ \\
\hline VB. 21 & $107 \pm 0.41$ & $99.83 \pm 0.40$ & $30.46 \pm 0.20$ & $26.13 \pm 0.78$ & $155 \pm 1.15$ & $87.33 \pm 0.33$ & $17.31 \pm 0.36$ & $13.84 \pm 0.26$ & $24.94 \pm 0.51$ & $17.49 \pm 0.10$ & $51.1 \pm 0.11$ & $37.30 \pm 0.60$ \\
\hline CSR 30 & $118.5 \pm 0.79$ & $109.1 \pm 0.41$ & $26.4 \pm 0.15$ & $23.66 \pm 0.63$ & $135 \pm 0.57$ & $93.33 \pm 0.33$ & $10.24 \pm 0.21$ & $6.18 \pm 0.21$ & $21.46 \pm 0.41$ & $16.12 \pm 0.14$ & $32.5 \pm 0.41$ & $23.53 \pm 0.60$ \\
\hline VB. 24 & $128.26 \pm 0.35$ & $116.96 \pm 0.49$ & $31.33 \pm 0.08$ & $27.16 \pm 0.66$ & $178.66 \pm 1.20$ & $108 \pm 0.57$ & $17.72 \pm 0.36$ & $10.72 \pm 0.31$ & $20.33 \pm 0.42$ & $16.39 \pm 0.24$ & $34.46 \pm 0.80$ & $23.33 \pm 0.54$ \\
\hline Type 3 & $126.9 \pm 0.30$ & $117 \pm 0.47$ & $27.46 \pm 0.14$ & $24.1 \pm 0.66$ & $168.66 \pm 0.88$ & $108 \pm 0.57$ & $17.05 \pm 0.35$ & $8.26 \pm 0.38$ & $21.7 \pm 0.45$ & $17.04 \pm 0.18$ & $40.93 \pm 0.98$ & $28.2 \pm 0.05$ \\
\hline Bas. 386 & $115.4 \pm 0.37$ & $105.13 \pm 0.41$ & $29.73 \pm 0.20$ & $25.06 \pm 0.92$ & $108 \pm 0.57$ & $57.71 \pm 0.29$ & $7.78 \pm 0.16$ & $2.97 \pm 0.33$ & $23.66 \pm 0.42$ & $16.42 \pm 0.18$ & $26.3 \pm 0.95$ & $17.03 \pm 0.57$ \\
\hline Pant Bas.-2 & $100.66 \pm 0.29$ & $92.1 \pm 0.37$ & $33.13 \pm 0.14$ & $28.63 \pm 0.70$ & $216.33 \pm 0.88$ & $129.33 \pm 0.33$ & $11.53 \pm 0.24$ & $5.54 \pm 0.38$ & $27.17 \pm 0.44$ & $18.97 \pm 0.28$ & $37.8 \pm 0.60$ & $29.8 \pm 0.55$ \\
\hline PB-1121 & $117.06 \pm 0.20$ & $113.16 \pm 0.49$ & $28.6 \pm 0.11$ & $26.53 \pm 0.24$ & $103.66 \pm 0.88$ & $80 \pm 0.57$ & $16.61 \pm 0.34$ & $14.13 \pm 0.24$ & $26.76 \pm 0.14$ & $22.69 \pm 0.24$ & $39.26 \pm 0.34$ & $32.26 \pm 0.63$ \\
\hline Pant Bas.-1 & $118.66 \pm 0.31$ & $108.93 \pm 0.41$ & $28.66 \pm 0.24$ & $24.7 \pm 0.43$ & $166.66 \pm 0.88$ & $97 \pm 0.577$ & $21.63 \pm 0.44$ & $10.77 \pm 0.26$ & $24.23 \pm 0.15$ & $18.8 \pm 0.13$ & $37.96 \pm 0.49$ & $27.5 \pm 0.28$ \\
\hline PS 5 & $107.5 \pm 0.41$ & $100.4 \pm 0.41$ & $32.96 \pm 0.63$ & $28.46 \pm 0.33$ & $128 \pm 0.57$ & $75.33 \pm 0.33$ & $14.93 \pm 0.31$ & $5.96 \pm 0.25$ & $24.71 \pm 0.14$ & $19.88 \pm 0.29$ & $42.2 \pm 0.5$ & $29.3 \pm 0.47$ \\
\hline VB. 22 & $98.16 \pm 0.44$ & $92.46 \pm 0.32$ & $28.63 \pm 0.24$ & $25.2 \pm 0.75$ & $153 \pm 0.57$ & $98.66 \pm 0.66$ & $14.3 \pm 0.29$ & $8.63 \pm 0.27$ & $21.3 \pm 0.18$ & $16.08 \pm 0.28$ & $33.63 \pm 0.61$ & $23.2 \pm 0.26$ \\
\hline C.D. & 1.56 & 1.21 & 0.78 & 1.72 & 2.44 & 2 & 0.91 & 0.8 & 1.05 & 0.63 & 1.82 & 1.69 \\
\hline SE(m) & 0.54 & 0.42 & 0.27 & 0.6 & 0.85 & 0.69 & 0.31 & 0.28 & 0.37 & 0.22 & 0.63 & 0.59 \\
\hline $\operatorname{SE}(d)$ & 0.77 & 0.60 & 0.38 & 0.85 & 1.2 & 0.98 & 0.45 & 0.39 & 0.52 & 0.31 & 0.9 & 0.83 \\
\hline C.V. & 0.85 & 0.71 & 1.64 & 4.12 & 0.99 & 1.27 & 3.69 & 5.37 & 2.67 & 2.1 & 3.14 & 3.95 \\
\hline
\end{tabular}

*C-Control, DS-Drought Stress 
Table.4 Morphological performance of rice genotypes under drought stress (2019-20)

\begin{tabular}{|c|c|c|c|c|c|c|c|c|c|c|c|c|}
\hline \multirow{2}{*}{$\begin{array}{l}\text { Name of } \\
\text { variety }\end{array}$} & \multicolumn{2}{|c|}{ Plant height } & \multicolumn{2}{|c|}{ Panicle length } & \multicolumn{2}{|c|}{ Seed per panicle } & \multicolumn{2}{|c|}{ Yield per plant } & \multicolumn{2}{|c|}{ Test weight } & \multicolumn{2}{|c|}{ Leaf area } \\
\hline & C & DS & C & DS & C & DS & C & DS & C & DS & C & DS \\
\hline Nagina-22 & $97.76 \pm 0.60$ & $95.5 \pm 0.47$ & $20.3 \pm 0.11$ & $18.86 \pm 0.17$ & $145.33 \pm 0.88$ & $123 \pm 1.15$ & $14.26 \pm 0.49$ & $12.55 \pm 0.40$ & $22.14 \pm 0.13$ & $20.07 \pm 0.39$ & $30.2 \pm 0.60$ & $25.73 \pm 0.57$ \\
\hline Panjab Bas.-04 & $92.73 \pm 0.49$ & $87.83 \pm 0.43$ & $23.3 \pm 0.15$ & $21.03 \pm 0.14$ & $120 \pm 0.57$ & $84 \pm 0.57$ & $15.85 \pm 0.15$ & $8.72 \pm 0.22$ & $26.43 \pm 0.14$ & $20 \pm 0.34$ & $25.3 \pm 1.12$ & $19.53 \pm 0.34$ \\
\hline Panjab Bas.-02 & $112.5 \pm 0.7$ & $103.86 \pm 0.60$ & $29.16 \pm 0.14$ & $25.26 \pm 0.12$ & $109.66 \pm 0.88$ & $66.66 \pm 0.33$ & $11.32 \pm 0.35$ & $5.66 \pm 0.41$ & $23.97 \pm 0.14$ & $17.42 \pm 0.30$ & $31.83 \pm 0.57$ & $23.53 \pm 0.57$ \\
\hline Pusa bas.-1609 & $104.33 \pm 0.66$ & $97.73 \pm 0.61$ & $30.16 \pm 0.23$ & $26.06 \pm 0.14$ & $214.33 \pm 0.88$ & $125.66 \pm 0.33$ & $16.83 \pm 0.32$ & $11.44 \pm 0.28$ & $28.70 \pm 0.15$ & $21.38 \pm 0.30$ & $54.5 \pm 0.60$ & $40.73 \pm 0.37$ \\
\hline VB-23 & $106.23 \pm 0.63$ & $99.06 \pm 0.58$ & $26.5 \pm 0.17$ & $24.03 \pm 0.14$ & $115.66 \pm 0.88$ & $83 \pm 0.57$ & $12.6 \pm 0.33$ & $8.56 \pm 0.24$ & $20.21 \pm 0.11$ & $14.80 \pm 0.62$ & $31.3 \pm 1.12$ & $24.13 \pm 0.57$ \\
\hline Pusa Bas.-1 & $115.73 \pm 0.69$ & $106.86 \pm 0.55$ & $30.16 \pm 0.20$ & $26.1 \pm 0.17$ & $181 \pm 0.57$ & $108 \pm 0.57$ & $16.66 \pm 0.53$ & $10.66 \pm 0.28$ & $22.96 \pm 0.12$ & $18.29 \pm 0.51$ & $30.5 \pm 0.60$ & $22.03 \pm 0.57$ \\
\hline Pusa Bas.-1509 & $97.5 \pm 0.47$ & $92.3 \pm 0.64$ & $26.6 \pm 0.11$ & $23.56 \pm 0.12$ & $95.33 \pm 0.88$ & $62 \pm 0.57$ & $16.28 \pm 0.57$ & $10.74 \pm 0.23$ & $25.89 \pm 0.17$ & $17.99 \pm 0.11$ & $33.1 \pm 1.18$ & $23.23 \pm 1.09$ \\
\hline Tarori Bas. & $129.5 \pm 0.78$ & $118.73 \pm 0.61$ & $28.73 \pm 0.14$ & $25.16 \pm 0.14$ & $119.66 \pm 0.88$ & $73 \pm 0.57$ & $14.54 \pm 0.30$ & $8.58 \pm 0.22$ & $24.333 \pm 0.13$ & $18.17 \pm 0.10$ & $25.2 \pm 0.60$ & $16.63 \pm 0.54$ \\
\hline Bas.-564 & $127.5 \pm 0.72$ & $116.33 \pm 0.63$ & $34.73 \pm 0.26$ & $30.1 \pm 0.17$ & $207 \pm 0.57$ & $122.33 \pm 0.33$ & $12.42 \pm 0.37$ & $6.71 \pm 0.35$ & $21.21 \pm 0.11$ & $15.88 \pm 0.38$ & $25.5 \pm 0.26$ & $19.26 \pm 0.53$ \\
\hline Pusa Bas.-6 & $92.5 \pm 0.58$ & $88.33 \pm 0.44$ & $28.33 \pm 0.20$ & $25.03 \pm 0.14$ & $155 \pm 0.57$ & $102.66 \pm 0.33$ & $18.38 \pm 0.47$ & $9.19 \pm 0.44$ & $22.21 \pm 0.11$ & $15.6 \pm 0.56$ & $36.3 \pm 0.32$ & $28.53 \pm 0.40$ \\
\hline VB.-21 & $107.33 \pm 0.63$ & $100.33 \pm 0.64$ & $30.5 \pm 0.23$ & $26 \pm 0.17$ & $157 \pm 0.57$ & $89.33 \pm 0.33$ & $17.22 \pm 0.67$ & $13.6 \pm 0.25$ & $25.15 \pm 0.16$ & $17.69 \pm 0.13$ & $51 \pm 0.43$ & $37.56 \pm 0.49$ \\
\hline Bas. CSR 30 & $118.73 \pm 0.66$ & $108.83 \pm 0.54$ & $26.3 \pm 0.17$ & $23.53 \pm 0.14$ & $136.66 \pm 0.33$ & $93.33 \pm 0.33$ & $10.62 \pm 0.44$ & $6.35 \pm 0.24$ & $21.25 \pm 0.11$ & $15.91 \pm 0.10$ & $32.73 \pm 0.41$ & $24.2 \pm 0.60$ \\
\hline VB-24 & $128.16 \pm 0.77$ & $116.2 \pm 0.58$ & $31.5 \pm 0.23$ & $27.16 \pm 0.17$ & $182 \pm 0.57$ & $108.66 \pm 0.66$ & $17.65 \pm 0.63$ & $10.59 \pm 0.30$ & $20.66 \pm 0.12$ & $16.46 \pm 0.50$ & $34.53 \pm 0.52$ & $23.53 \pm 0.61$ \\
\hline Type-3 & $126.73 \pm 0.72$ & $117.43 \pm 0.61$ & $27.5 \pm 0.17$ & $24.13 \pm 0.17$ & $172.33 \pm 0.33$ & $109 \pm 0.57$ & $17.13 \pm 0.51$ & $8.39 \pm 0.27$ & $22.11 \pm 0.12$ & $17.32 \pm 0.24$ & $41 \pm 0.52$ & $28.33 \pm 0.46$ \\
\hline Bas.-386 & $115.63 \pm 0.76$ & $104.86 \pm 0.51$ & $29.5 \pm 0.17$ & $24.96 \pm 0.21$ & $105 \pm 0.57$ & $57 \pm 0.57$ & $7.82 \pm 0.55$ & $3.10 \pm 0.42$ & $23.75 \pm 0.13$ & $16.23 \pm 0.30$ & $26.33 \pm 0.69$ & $17.20 \pm 0.60$ \\
\hline Pant Bas.-2 & $100.5 \pm 0.60$ & $92.2 \pm 0.58$ & $31.33 \pm 0.20$ & $27.1 \pm 0.17$ & $215.33 \pm 0.33$ & $128.66 \pm 0.66$ & $11.61 \pm 0.61$ & $5.57 \pm 0.41$ & $26.66 \pm 0.15$ & $18.57 \pm 0.31$ & $37.5 \pm 0.60$ & $29.93 \pm 0.46$ \\
\hline PB-1121 & $117.3 \pm 0.64$ & $113.23 \pm 0.56$ & $28.76 \pm 0.17$ & $26.6 \pm 0.11$ & $107.33 \pm 0.33$ & $83.33 \pm 0.33$ & $16.24 \pm 0.28$ & $13.8 \pm 0.20$ & $26.75 \pm 0.15$ & $22.65 \pm 0.27$ & $39.53 \pm 0.63$ & $32.43 \pm 0.54$ \\
\hline Pant Bas.-1 & $118.5 \pm 0.75$ & $108.63 \pm 0.54$ & $28.5 \pm 0.17$ & $26.06 \pm 0.17$ & $167.66 \pm 0.33$ & $97 \pm 0.57$ & $21.66 \pm 0.65$ & $10.81 \pm 0.33$ & $24.33 \pm 0.14$ & $16.85 \pm 0.35$ & $38.3 \pm 0.60$ & $27.53 \pm 0.80$ \\
\hline PS-5 & $107.63 \pm 0.56$ & $100.36 \pm 0.53$ & $32.76 \pm 0.23$ & $28.26 \pm 0.20$ & $123 \pm 0.57$ & $72 \pm 0.57$ & $15.21 \pm 0.59$ & $6.08 \pm 0.27$ & $24.75 \pm 0.14$ & $19.72 \pm 0.43$ & $41.83 \pm 0.60$ & $29.36 \pm 0.52$ \\
\hline VB.-22 & $98.33 \pm 0.52$ & $92.1 \pm 0.55$ & $28.5 \pm 0.17$ & $25.13 \pm 0.26$ & $159 \pm 0.57$ & $101 \pm 0.57$ & $14.53 \pm 0.65$ & $8.72 \pm 0.47$ & $21.66 \pm 0.12$ & $16.35 \pm 0.26$ & $33.83 \pm 0.43$ & $23.23 \pm 0.54$ \\
\hline C.D. & 1.88 & 1.63 & 0.54 & 0.48 & 1.83 & 1.61 & 1.43 & 0.93 & 0.39 & 1.03 & 1.92 & 1.68 \\
\hline SE(m) & 0.65 & 0.56 & 0.18 & 0.16 & 0.64 & 0.56 & 0.49 & 0.32 & 0.13 & 0.36 & 0.67 & 0.58 \\
\hline $\operatorname{SE}(d)$ & 0.92 & 0.8 & 0.26 & 0.23 & 0.9 & 0.79 & 0.7 & 0.46 & 0.19 & 0.5 & 0.94 & 0.82 \\
\hline C.V. & 1.02 & 0.95 & 1.13 & 1.16 & 0.74 & 1.03 & 5.78 & 6.28 & 1.01 & 3.49 & 3.32 & 3.93 \\
\hline
\end{tabular}

*C-Control, DS-Drought Stress 
Graph.2 Percent reduction of traits under drought stress (2018-19)

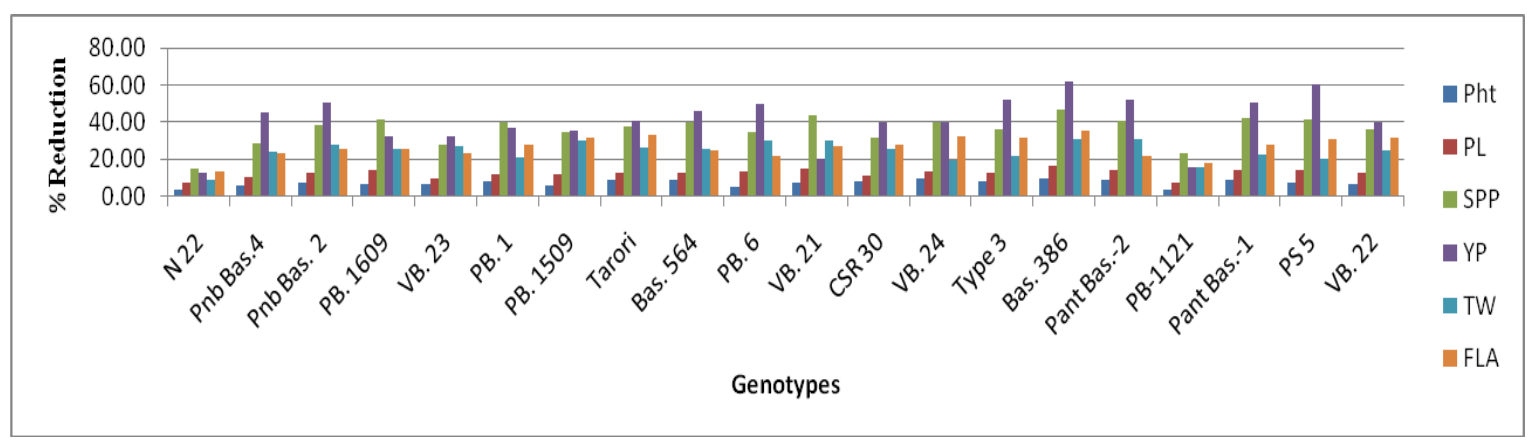

Graph.3 Percent reduction of traits under drought stress (2019-20)

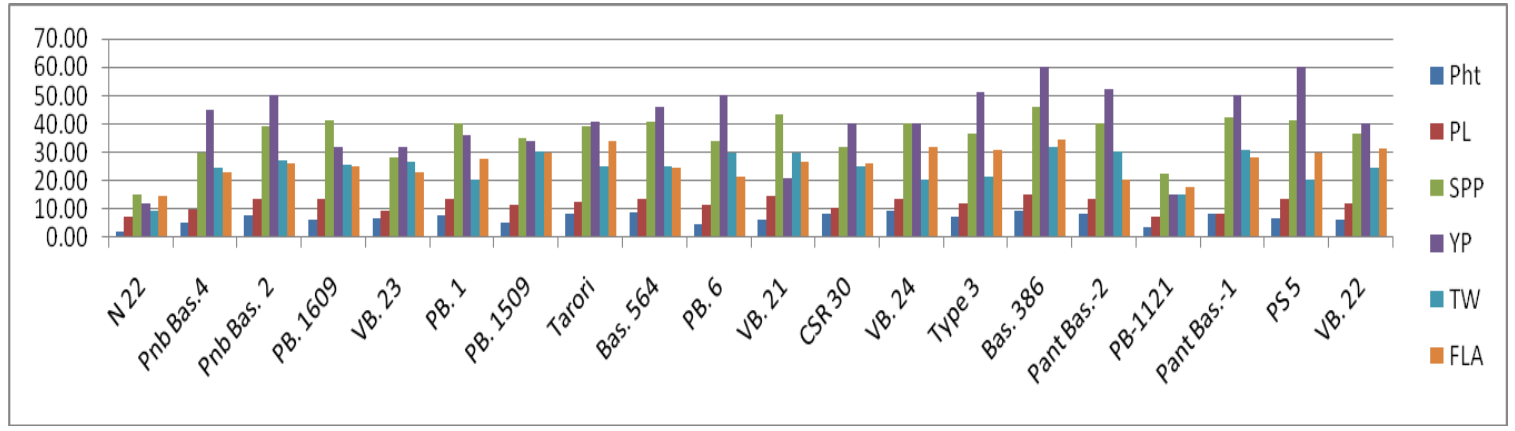

Water stress significantly affected the plant height at booting, flowering and grain filling stage in compare to control plants. In present study, the minimum difference was observed in genotype Nagina $22(2.31 \%)$ and maximum difference was observed in genotype Basmati $364(9.31 \%)$ for both years. This outcome agrees with Islam et al., (1994), who found that below 20 percent soil saturation at booting and flowering phases, moisture stress decreased plant height. Similar findings have also been reported by Ahmadikhah and Marufinia (2016). The reduction in height might be either due to inhibition of cell length or cell division by water deficits. Rahman et al., (2002) also reported that there were remarkable differences in plant height among the varieties under water stress. They observed with compare to control plants, the plant height decreases under soil moisture stress (30\% of field capacity), ranges from $2.30 \%$ to $9.41 \%$.
In present study, the minimum difference was observed in genotype Nagina $22(7.06 \%)$ and the maximum difference was observed in genotype Basmati 386 (15.69\%) for both years. Due to water stress, the length of panicles was significantly reduced in all genotypes in relative to control plants (Rahman et al., 2002). Ekanayake (1987) and Islam et al., (1994) also founds the similar results. This might be due to the fact that moisture stress reduced the synthesis of carbohydrate and or weaken the sink at reproductive stages. Rahman et al., (2002) reported that there were remarkable differences on panicle length among the varieties under stress at flowering time. With compare to control plants, the length of panicle decreases under soil moisture stress ( $30 \%$ of field capacity), ranges from $2.46 \%$ to $8.87 \%$.

Water stress decreased the number and weight of grains at the reproductive and grain-filling 
stage. When drought stress occurred at flowering, the total grain number per panicle was significantly reduced (Sarvestani et al., 2008). In present study, the minimum difference was observed in genotype Nagina $22(14.74 \%)$ and the maximum difference was observed in genotype Basmati 386 (46.56\%) for both years. This indicated the reduced crop growth at flowering time due to drought. Rahman et al., (2002) and Islam et al., (1994) also observed the number of filled grains per panicle decreased significantly compared to control with the moisture stress at booting, flowering and grain filling phases. They observed the lowest (71.24) number of filled grains per panicle during stress at flowering stage. Yang et al., 2019, recorded that the number of spikelets per panicle (SPN) and filled grains (FG) yield components were significantly reduced by 18 percent and 19 percent for YLY6 and 21 percent and 19 percent for HY113, respectively, compared to the conventional flooding irrigation system on average over two seasons, leading to the significant decrease in GY in YLY6 and HY111 under stress condition.

Water stress at grain filling (anthesis to maturity) was more detrimental followed by panicle initiation stage regarding filled grains/panicle, effective tillers/hill, total spikelets/panicle, 1000-grain weight and grain yield/hill, irrespective of the genotypes (Moonmoon and Islam, 2017). The yield depends on dry matter accumulation and its partitioning (Baruah et al., 2006). Rice grain yield can be restricted by the availability of grain-developing assimilates (source limitation) or by the reproductive organ's ability to accept assimilates (sink capacity). Yield losses from the usual level due to water deficit are helpful in assessing drought tolerance in as much as cultivar differs greatly in inherent yielding capacity (Pirdashti et al., 2004). Pantuwan et al., (2002) and Cattivelli et al., (2008) have recorded decreased rice yield at critical growth stages due to drought stress. In present study, the minimum difference in yield was observed Nagina 22 $(12.03 \%)$ and the maximum difference was observed in genotype Basmati 386 (61.83\%) for both years. These findings are similar with some researchers who stated that if drought occurs during flowering time, grain yield could be dramatically decreased (about 60\%) (Boonjung and Fukai 1996). Similarly, Rahman et al., (2002) reported decreases in grain yield per hill under soil water stress (30 percent of field capacity), ranging from 16.07 percent to 41.21 percent. Yang et al., (2019) also observed that the grain yield (GY) under drought stress was significantly reduced for YLY6 by 23.2 percent and for HY113 by 24.0 percent compared to traditional flooding.

The reduction in yield at the flowering stage under drought stress was mainly due to a decrease in the total number of grains per panicle (increase in unfilled grain and a greatly decreased proportion of filled grains) and 1000-grain weight, respectively. Islam (1999) and Islam et al., (1994) have demonstrated similar findings on 1000-grain weight under water stress at booting and flowering stages. In present study, the minimum difference was observed in genotype Nagina $22(8.46 \%)$ and maximum difference was observed in genotype Basmati $386(31.66 \%)$ for both years. Sarvestani et al., 2008 reported that the drought stress at grain filling stage reduced the 1000-grain weight by $17 \%$ than control. In terms of 1000 - grain weight, mentioned cultivars show a reduction under non-submerged treatment, however the reduction was higher in 829 cultivar $(8.1 \%)$ (Mostajeran and Rahimi-Eichi, 2009). Similar results on 1000-grain weight under water stress had been reported by Venuprasad et al., (2007) and Castillo et al., (2006). Rahman et al., (2002) reported that there were remarkable differences in 1000 grain weight among the varieties under stress at flowering 
time. With compare to control plants, the 1000 grain weight decreases under soil moisture stress (30\% of field capacity), ranges from $1.07 \%$ to $7.80 \%$. Stress could decrease translocation of assimilates to the grains during different growth stages, which decreased gram weight and increased the empty grains. The efficient translocation of assimilates towards sink can be indicated $\mathrm{HI}$ values. Lower $\mathrm{HI}$ values at booting and flowering stages under stress suggest that the translocation of assimilates to grains over grains filling stage was more detrimental (Rahman et al., 2002). Under extreme drought stress, it was argued that when yields are decreased to below $50 \%$ of those wider favorable conditions the relationship between yield under favorable and stress conditions break down (Ceccarelli and Grando, 1991). The results of this experiment indicate that under these circumstances, genotypes were not able to convey their genetic yield potential.

Leaf area of rice genotypes significantly reduced irrespective of genotypes. In present study, the minimum difference was observed in genotype Nagina $22(13.26 \%)$ and the maximum difference was observed in genotype Basmati 386 (35.24\%) for both years. Similarly, the high reduction in leaf area was recorded in Swarna Sub 1 (49.08\%) while low in Nagina 22(18.70\%) Susk Samrat (28.22\%), NDR 97 (22.40\%) and NDR 102 (20.60\%) (Singh et al., 2018). Negative effects of water deficiency on mineral nutrition and metabolism reduce the leaf area and change the partitioning of plant organs (Zain et al., 2014).

In conclusion, all the morphological parameters was found significantly reduced under drought stress condition at flowering stage and directly makes a negative impact on yield of rice genotypes .

\section{Acknowledgements}

This study was supported by Sardar Vallabhbhai Patel University of Agriculture and Technology, Modipuram, Meerut. The authors of the manuscript are highly grateful to Indian Council of Agricultural Research, New Delhi, India, to provide the financial support in the form of Indian Council of Agricultural Research-Senior Research Fellowship.

\section{Conflicts of interest}

Authors declare no conflicts of interest.

\section{References}

Ahmadikhah, A., and Marufinia, A. (2016). Effect of reduced plant height on drought tolerance in rice. Biotech, 6(221): 1-9.

Baruah, K.K., Rajkhowa, S.C. and Das K. (2006). Physiological analysis of growth, yield development and grain quality of some deep water rice cultivars. Agron and Crop Science. 192: 228-232.

Beck, EH., Fettig, S., Knake, C., K. and Bhattarai T. (2007). Specific and unspecific responses of plants to cold and drought stress. Bioscience. 32:501-510.

Boonjung, H. and Fukai, S. (1996). Effects of soil water deficit at different growth stages on rice growth and yield under upland conditions. Field Crops Research, 48: 47-55. http://dx.doi.org/10.1016 /0378-4290(96)00039-1

Bouman, B. A. M., Peng, S., Castaneda, AR. and Visperas R. M. (2005). Yield and water use of irrigated tropical aerobic rice systems. Agric Water Manag. 74: 87-105.

Castillo, E.G., Tuong, T.P., Singh, U., Inubushi, K., and Padilla, J. (2006). Drought response of dry-seeded rice to water stress timing and $\mathrm{N}$-fertilizer rates and sources. Soil Science and Plant Nutrition, 52(4): 496-508.

Cattivelli, L., Rizza, F., Badeck, F. W., Mazzucotelli, E., Mastrangelo, A. M., 
Francia, E., Mare, C., Tondelli, A. and Stanca, M. (2008). Drought tolerance improvement in crop plants: An integrated view from breeding to genomics. Field Crops Res, 105:1-14.

Ceccarelli, S. and Grando, S. (1991). Selection environment and environmental sensitivity in barley. Euphytica 57: 157167.

Chaturvedi, G.S. and Ingram, K.T. (1988). Assimilate translocation of lowland rice in response to water deficit and shad. Philippine Journal of Crop Science. 13 (1):55-59.

FAOSTAT. (2018-19). http://www.fao.org/faostat/en/\#data/QC

Farooq, M., Wahid, A., Kobayashi, N., Fujita, D., and Basra, S. M. A. (2009b). Plant drought stress: effects, mechanisms and management. Agron. Sustain. Dev., 29: 185-212. doi: 10.1051/agro:2008021

Fujita, M., Fujita, Y., Noutoshi, Y., Takahashi, F., Narusaka, Y., Yamaguchi-Shinozaki, K., et al., (2006). Crosstalk between abiotic and biotic stress responses: a current view from the points of convergence in the stress signaling networks. Curr. Opin. Plant Biol., 9: 436442. doi: 10.1016/j.pbi.2006.05.014

Hsiao, T. C., Toole, J. C., Yambao, E. B. and Turner, N. C. (1984). Influence of osmotic adjustment on leaf rolling and tissue death in rice (Oryza sativa L.). Plant Physiol., 75: 338-341.

Islam, M. T., Salam, M.A. and Kauser, M. (1994). Effect of soil water stress at different growth stages of rice of yield components and yield. Progress. Agric., 5(20): 151-156.

Khush G. (2005). What it will take to Feed 5.0 Billion Rice Consumers in 2030. Plant Molecular Biology, 59:1-6.

Maisura, Chozin., M. A, Lubis., I., Junaedinand, A. and Ehara, H. (2014). Some physiological character responses of rice under drought conditions in a paddy system. Journal of International Society of Southeast Asian Agricultural Science. 20(1): 104-114.
Mishra, B.K. (2005). Histochemical studies on accumulation and remobilization of starch at flowering stage in upland rices. M.Sc. Thesis, Narendra Deva University of Agriculture of Agriculture and Technology, Narendra Nagar, Kumarganj, Faizabad, U.P., India.

Mostajeran A. and Rahimi V. (2009). Effects of Drought Stress on Growth and Yield of Rice (Oryza sativa L.) Cultivars and accumulation of proline and soluble sugars in sheath and blades of their different ages leaves. American-Eurasian J. Agric. \& Environ. Sci., 5 (2): 264-272.

Nachimuthu, V. V., Muthurajan, R., Duraialaguraja, S., Sivakami, R., Pandian, B. A. and Ponniah G. (2015). Analysis of population structure and genetic diversity in rice germplasm using SSR Markers: An initiative towards association mapping of agronomic traits in Oryza sativa. Rice. 8:30-54.

O'Toole, J. C. and Cruz, R. T. (1983). Genotypic variation in epicuticular wax of rice. Crop Science. 23: 393-394.

O'Toole, J. C. (1982). Adaptation of rice to drought- prone environments. In: Drought resistance in crops with the emphasis on rice. Manila: IRRI, 195-213.

Pandey, V. and Shukla, A. (2015). Acclimation and tolerance studies in rice under drought stress. Rice Science. 22(4): 147161.

Pantuwan, G., Fukai, S., Cooper, M., Rajatasereekul, S. and O'Toole, J. C. (2002). Yield response of rice (Oryza sativa L.) genotypes to drought under rainfed lowlands. 2. Selection of drought resistant genotypes. Field Crops Res., 73:169-180. doi: 10.1016/S03784290(01)00195-2.

Passioura, J. B. (1996). "Drought and Drought Tolerance". Plant Growth Regulation, 20(2): 79-83.

Passioura, J. B. (2007). The drought environment: physical, biological and agricultural perspectives. Journal of Experimental Botany, 58:113-117.

Pirdashti, H., Sarvestani, Z. and Bahmanyar, M. 
(2009). Comparison of physiological responses among four contrast rice cultivars under drought stress conditions. World Academy of Science, Engineering and Technology, 49:52-53.

Pirdashti, H., Tahmasebi, S. Z. and Nematza, D. G. (2004). Study of water stress effects in different growth stages on yield and yield components of different rice cultivars. 4 th International Crop Science Congress, Brisbane, Australia.

Rahman, M.T., Islam, M. T. and Islam, M. O. (2002). Effect of water stress at different growth stages on yield and yield contributing characters of transplanted Aman rice. Pak. J. Biol. Sci., 5(2): 169172.

Sarvestani, Z.T., Pirdashti, H., Sanavy, S.A. and Balouchi, H. (2008). Study of water stress effects in different growth stages on yield and yield components of different rice (Oryza sativa L.) cultivars. Pakistan Journal of Biological Science, 11(10): 1303-1309.

Singh, S., Prasad, S., Yadav, V., Kumar, Ajay., Jaiswal, Bandana., Kumar, Adesh., Khan, N.A., and Dwivedi, D. K. (2018). Effect of drought stress on yield and yield components of Rice (Oryza sativa L.) genotypes. Int.J.Curr.Microbiol.App.Sci. Special Issue-7: 2752-2759

Szekeres M. (2003). Brassinosteroid and system in: two hormones perceived by the same receptor. Trends Plant Sci., 8:102-104.

Venuprasad, R., Cruz, M. T., Amante, M., Magbanua, R., Kumar, A. and Atlin, G. N. (2008). Response to two cycles of divergent selection for grain yield under drought stress in four rice breeding populations. Field Crops Research, 107:232-244.

Venuprasad, R., Lafitte, H. R. and Atlin, G. N. (2007). Response to direct selection for grain yield under drought stress in rice. Crop Science, 47(1): 285-293.

Yang, X, Wang, B., Chen, L., Li, P. and Cao., C. (2019). The different influences of drought stress at the flowering stage on rice physiological traits, grain yield, and quality. Scientific Reports, 9(3742).

Yang, P.M., Huang, Q.C., Qin, G.Y., Zhao, S.P. and Zhou, J.G. (2014). Different droughtstress responses in photosynthesis and reactive oxygen metabolism between autotetraploid and diploid rice. Photosynthetica. 52(2): 193-202.

Zain, N. A. M., Ismail, M. R., Puteh, A., Mahmood, M. and Islam, M. R. (2014). Drought tolerance and ion accumulation of rice following application of additional potassium fertilizer, Communications in Soil Science and Plant Analysis, DOI: 10.1080/00103624.2014.932374.

\section{How to cite this article:}

Arun Kumar, R. S. Sengar, Manoj Yadav, Shalani Gupta, Ramji Singh and Pooran Chand. 2020. Impact of Flowering Stage Drought Stress on Yield and Yield Related Attributes on Rice (Oryza sativa L) Genotypes. Int.J.Curr.Microbiol.App.Sci. 9(12): 1823-1836. doi: https://doi.org/10.20546/ijcmas.2020.912.217 